TITLE:

\title{
Characterization of non-Gaussian mid-infrared free-electron laser beams by the knife-edge method
}

\section{AUTHOR(S):}

Qin, Yu; Nakajima, Takashi; Zen, Heishun; Wang,

Xiaolong; Kii, Toshiteru; Ohgaki, Hideaki

\section{CITATION:}

Qin, Yu ... [et al]. Characterization of non-Gaussian mid-infrared free-electron laser beams by the knife-edge method. Infrared Physics \& Technology 2014, 66: 146-151

\section{ISSUE DATE:}

2014-09

URL:

http://hdl.handle.net/2433/189382

\section{RIGHT:}

(C) 2014 Elsevier B.V.; This is not the published version. Please cite only the published version.; この論文は出版社版でありません。引用の際に は出版社版をご確認ご利用ください。 


\title{
Characterization of non-Gaussian mid-infrared free-electron laser beams by the knife-edge method
}

\author{
Yu Qin, Takashi Nakajima, Heishun Zen, Xiaolong Wang, Toshiteru Kii, \\ Hideaki Ohgaki \\ Institute of Advanced Energy, Kyoto University, Gokasho, Uji, Kyoto 611-0011, Japan
}

\begin{abstract}
We report the characterization of mid-infrared free-electron laser (FEL) beams at the wavelength of $11 \mu \mathrm{m}$ by the knife-edge method. From the knife-edge data we find that the FEL beam has a non-Gaussian shape. To represent the non-Gaussian beam shape we employ two methods: fitting the knife-edge data to some analytical functions with a few free parameters and numerical smoothing of the knife-edge data. Both methods work equally well. Using those data we can reconstruct the two-dimensional (2D) beam profiles at different positions around the focus by assuming that the $2 \mathrm{D}$ intensity distribution function is separable in $\mathrm{x}$ (horizontal) and $\mathrm{y}$ (vertical) directions. Using the 2D beam profiles at different positions around the focus, we find that the beam propagation factor $\left(M^{2}\right.$ factor) is $\sim 1.1$ in both $\mathrm{x}$ and $\mathrm{y}$ directions. As a cross-check, we also carry out the burn pattern experiment to find that the behavior of the focused FEL beam along the propagation is consistent with the results obtained by the knife-edge method.
\end{abstract}

Keywords: Free-Electron Laser, Non-Gaussian beam, Mid-infrared, knife-edge method, $M^{2}$ measurement

\section{INTRODUCTION}

Since its first lasing in 1976 [1], FELs have attracted a lot of interests in various research areas due to their high power and large wavelength tunablities [2]. The FEL we have at our institute, Kyoto University free-electron

Email addresses: nakajima@iae.kyoto-u.ac.jp (Takashi Nakajima), ohgaki@iae.kyoto-u.ac.jp (Hideaki Ohgaki ) 
laser (KU-FEL), is an oscillator-type FEL at the mid-infrared wavelength of $5-13 \mu \mathrm{m}$ with the macropulse repetition rate of $1 \mathrm{~Hz}$ [3]. Each macropulse has a duration of $\sim 1.5 \mu$ s and contains several thousands of micropulses with a duration of $\sim 0.6 \mathrm{ps}$ and an interval of $350 \mathrm{ps}$ between them. For many applications such as mid-infrared spectroscopy and nonlinear optics, it is crucial to have the knowledge on the micropulse duration, wavelength stability, and the spatial beam quality. For this reason we have recently measured the micropulse duration and wavelength stability of KU-FEL at $12 \mu \mathrm{m}$ under the presence of unknown amount of chirp by a new method, which is a variant of the fringe-resolved autocorrelation [4], and the single-shot spectra of temporally selected micropulses from KU-FEL at $11 \mu \mathrm{m}$ using the sum-frequency mixing technique [5]. Most recently, we have demonstrated that the KU-FEL pulses gated by a plasma mirror with unusually long (nanosecond) switching pulses have the high focusability [6], which results in nonlinear spectral broadening by focusing the beam into the nonlinear target. Although the fact that we have observed the nonlinear spectral broadening clearly implies that the high intensity has been achieved upon focusing, we still do not know the spatial beam quality of the incident FEL beam.

To measure the spatial beam quality, the most straightforward way is to use a commercial $M^{2}$ analyzer. Unfortunately there is no commercial $M^{2}$ analyzers available for the beam at the wavelength of $>1.8 \mu \mathrm{m}$, in particular with a very low repetition rate. The second choice is to use a $2 \mathrm{D}$-array detector (beam profiler) and measure the beam profiles at different positions along the propagation. However, one must carry out a detailed analysis by themselves, since the commercial beam profilers do not have the function to determine the $M^{2}$ value. The above two methods are rather expensive (more than 20,000 USD), and hence such convenient commercial devices are not always available to the FEL users. The third choice is to build a device by ourselves based on more conventional methods such as slit scan [7], pinhole scan [8], and knife-edge scan [9, 10]. Among these techniques, the knife-edge method is most commonly used due to high signal-to-noise ratio and excellent spatial resolution. Indeed, the knife-edge measurements have been performed for the oscillator-type FELs in a continuous pulse-train mode $[11,12]$. If better accuracy is desired, one can carry out a tomographic beam profile measurement by the knife-edge scan in many (usually more than 7) directions, and reconstruct the $2 \mathrm{D}$ beam profile using the inverse Radon transform [13].

In this paper, we report the characterization of non-Gaussian mid-infrared 
$(\sim 11 \mu \mathrm{m})$ FEL beam by the $2 \mathrm{D}$ knife-edge method. Known the fact that analytical methods developed for Gaussian beams under the knife-edge measurement $[10,14,15]$ do not work, the new ingredient in this work is the detailed report of the data analysis for non-Gaussian beam, which would be useful not only in the mid-IR but also in the other wavelength range such as extreme ultraviolet and x-ray.

For the data analysis, we employ two methods to represent non-Gaussian beam shape. One is fitting method to represent the real beam shape by some simple analytical function with a few free parameters to be fitted, and the other is smoothing method so that the derivatives of the knife-edge signals can be smoothly represented. It turns out that both methods work equally well, and we can reconstruct the $2 \mathrm{D}$ beam profiles by taking the derivatives of the knife-edge signals under the assumption that the 2D intensity distribution function is separable in two (horizontal and vertical) directions. Then, by making use of the $2 \mathrm{D}$ beam profiles reconstructed at different positions around the focus, we can obtain the variation of the beam diameter around the focus. Again, special attention has to be paid to deduce the beam diameter from the 2D beam profiles due to non-Gaussian feature of KU-FEL beams. Finally we deduce the $M^{2}$ factors to be about 1.1 in both x and y directions. As a cross-check, we also carry out the burn pattern measurement, and find that the burn patterns are consistent with the results by the knife-edge method. We note that the ablative imprints, which are similar to the burn patterns but with three-dimensional information by atomic force microscopy, have been used to characterize the spot size of X-ray laser beams [16]. More sophisticated online diagnostics system using extreme ultraviolet Hartmann sensors has been developed for FLASH [17].

\section{EXPERIMENTAL SETUP}

The experimental setup for knife-edge measurements is shown in Fig. 1(a) for the case of $\mathrm{x}$ direction scan, where $\mathrm{x}, \mathrm{y}$, and $\mathrm{z}$ axes stand for the horizontal, vertical, and the laser propagation directions, respectively. The laser beam from the output of the KU-FEL with the beam diameter of $\sim 14 \mathrm{~mm}$ (for $1 / e^{2}$ ) at the central wavelength of $11 \mu \mathrm{m}$ is focused by a $f=150 \mathrm{~mm}$ ZnSe lens. A knife-edge mounted on a three-axis translational stage is placed in the xy plane near the focus with the edge oriented to the y direction. The energy of the FEL pulse transmitted past the knife-edge is measured by the signal detector (Gentec. EO, QE8SP-I-BL-BNC), which we call $E_{\text {sig. The }}$ 
(a)

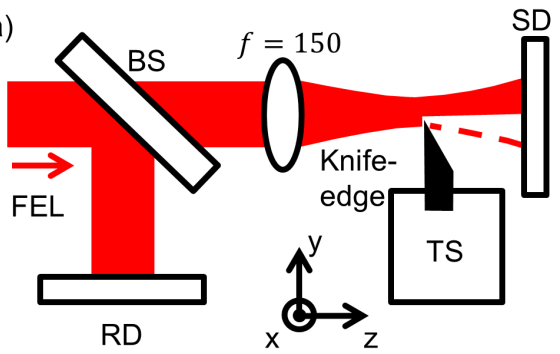

(b)

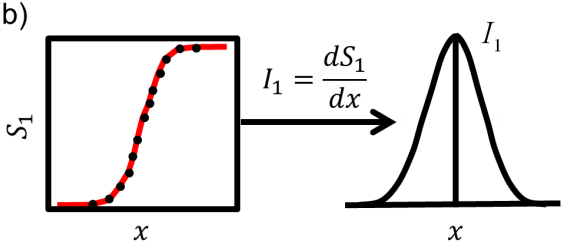

Figure 1: (a) Experimental setup (top view). The BS, SD, RD, and TS stand for the beam splitter, signal detector, reference detector and translational stage, respectively. (b) Illustration of the transmitted energy $S_{1}$ as a function of $x$ and its derivative.

shot-to-shot fluctuation of macropulse energy is typically $\pm 16 \%$ during the measurement, and it is not very different from the normal distribution. To reduce the influence of shot-to-shot fluctuation of FEL pulse energy, we monitor the FEL pulse energy before the lens with a reference detector (Newport, 818E-10-50-S), which we call $E_{\text {ref }}$. After averaging over 20 shots at each position we record the normalized transmitted energy $S_{1}=E_{\text {sig }} / E_{\text {ref }}$ during the scan in x direction. For the central scan range where $S_{1}$ changes from $20 \%$ to $80 \%$ with respect to that of the unblocked beam, the step size is 10 $\mu \mathrm{m}$, while at the beginning and end of the scan range, the step size is 20 $\mu \mathrm{m}$. Figure 1(b) illustrates the shape of $S_{1}$ (left) and its derivative, $d S_{1} / d x$ (right). We repeat the similar measurement at 8 different positions along the $\mathrm{z}$ direction around the focus. We also carry out the similar knife-edge measurements in y direction, while the normalized transmitted energy is called $S_{2}$.

It is known that FELs, in particluar in a pulsed-mode, may exhibit nonnegligible shot-to-shot pointing jitter, which may influence the results of the knife-edge measurement. During the data acquisition of $S_{1}\left(S_{2}\right)$ for a given position defined by $x_{n}\left(y_{n}\right)$ and $z$, we find that the shot-to-shot differences of the values of $S_{1}\left(x_{n}\right)\left(S_{2}\left(y_{n}\right)\right)$ are almost the same for all $x_{n}\left(y_{n}\right)$ 's, and take the value of $\sim 0.05$, while $S_{1}\left(S_{2}\right)$ is $\sim 2$ when the knife cut half of the beam (see Fig. 3). To estimate the pointing jitter and its influence on the knife-edge 
measurement, the largest values of $\left|S_{1}\left(x_{n}\right)-S_{1}\left(x_{n+1}\right)\right|$ and $\left|S_{2}\left(y_{n}\right)-S_{2}\left(y_{n+1}\right)\right|$ are listed in Table 1 for different $z$, when the transmitted energy is between 20 $\sim 80 \%$. Note that the step size for this range is $10 \mu \mathrm{m}$, i.e., $x_{n+1}-x_{n}=10 \mu \mathrm{m}$. Needless to say $\left|S_{1}\left(x_{n}+1\right)-S_{1}\left(x_{n}\right)\right|\left(\left|S_{2}\left(y_{n}+1\right)-S_{2}\left(y_{n}\right)\right|\right)$ becomes the largest value when the knife cut nearly half of the beam (see Fig. 1(b)).

Table 1: Largest values of $\left|S_{1}\left(x_{n+1}\right)-S_{1}\left(x_{n}\right)\right|$ and $\left|S_{2}\left(y_{n+1}\right)-S_{2}\left(y_{n}\right)\right|$ for different z.

\begin{tabular}{ccc}
\hline$z(\mathrm{~mm})$ & $\max \left[\left|S_{1}\left(x_{n}+1\right)-S_{1}\left(x_{n}\right)\right|\right]$ & $\max \left[\left|S_{2}\left(y_{n}+1\right)-S_{2}\left(y_{n}\right)\right|\right]$ \\
\hline 150 & 0.10 & 0.15 \\
154 & 0.28 & 0.25 \\
155 & 0.30 & 0.30 \\
156 & 0.35 & 0.42 \\
158 & 0.42 & 0.45 \\
160 & 0.30 & 0.35 \\
162 & 0.23 & 0.23 \\
166 & 0.12 & 0.13
\end{tabular}

From Table 1 , we find that even when the beam diameter is relatively large, i.e., $\sim 700 \mu \mathrm{m}$ at $z=150$ and $166 \mathrm{~mm}$ (see Fig. 8), the shot-to-shot differences of the values of $S_{1}\left(x_{n}\right)\left(S_{2}\left(y_{n}\right)\right)$, which is $\sim 0.05$, is smaller than the largest value of $\left|S_{1}\left(x_{n+1}\right)-S_{1}\left(x_{n}\right)\right|\left(\left|S_{2}\left(y_{n+1}\right)-S_{2}\left(y_{n}\right)\right|\right)$ at the same $z$. This implies that we will not miss the peak of $d S_{1} / d x\left(d S_{2} / d y\right)$ even under the presence of shot-to-shot change of the value of $S_{1}\left(S_{2}\right)$, whether the origin of such fluctuations is beam pointing jitter or not. We can also justify that our choice of the step size, $10 \mu \mathrm{m}$, is reasonable. Based on the above argument, we can say that the pointing jitter is at most $10 \mu \mathrm{m}$ if the beam diameter is $\sim 700 \mu \mathrm{m}$ (at $z=150$ and $166 \mathrm{~mm}$ ), or at most $1.4 \%$ of the beam diameter. For such a small jitter, taking the average of the data by multiple laser shots should be reasonable.

\section{DATA ANALYSIS}

\subsection{Reconstruction of the $2 D$ beam profile}

\subsubsection{Methodology}

We introduce $I(x, y)$ to represent the 2D intensity distribution of the FEL beam. During the scan in $\mathrm{x}$ direction, the normalized energy of the transmitted beam changes as a function of $x$, i.e., 


$$
S_{1}(x) \propto \int_{-\infty}^{x} \int_{-\infty}^{\infty} I\left(x^{\prime}, y\right) d x^{\prime} d y
$$

Now we assume that the 2D intensity distribution function is separable in $\mathrm{x}$ and y directions. Then,

$$
I(x, y)=I_{1}(x) \times I_{2}(y),
$$

where $I_{1}$ and $I_{2}$ are the intensity distribution functions of the laser beam along $\mathrm{x}$ and $\mathrm{y}$ directions, respectively. Under this assumption Eq. (1) is rewritten as

$$
S_{1}(x) \propto \int_{-\infty}^{\infty} I_{2}(y) d y \times \int_{-\infty}^{x} I_{1}\left(x^{\prime}\right) d x^{\prime} \propto \int_{-\infty}^{x} I_{1}\left(x^{\prime}\right) d x^{\prime} .
$$

Equation (3) says that $S_{1}$ is nothing but the integration of $I_{1}$ over x. Therefore, by taking the derivative of $S_{1}$, we can obtain the intensity distribution function along x direction. It reads

$$
I_{1}(x) \propto \frac{d S_{1}}{d x} .
$$

In Fig. 1(b), we illustrate $S_{1}$ and $I_{1}$. We can obtain $I_{2}$ in a similar way. Once we obtain the intensity distribution functions along both $\mathrm{x}$ and $\mathrm{y}$ directions, namely $I_{1}(x)$ and $I_{2}(y)$, we can reconstruct the $2 \mathrm{D}$ beam profile using Eq. (2).

To test the validity of our assumption on the separability of $I(x, y)$ into $I_{1}(x)$ and $I_{2}(y)$, we do the numerical experiments by assuming four different incident beam profiles, i.e., radially symmetric Gaussian beam, elliptical Gaussian beam, tilted elliptical Gaussian beam, and radially symmetric sech ${ }^{2}$ beam, and numerically scan the knife-edge in $\mathrm{x}$ as well as y directions to obtain $S_{1}$ and $S_{2}$, from which we can calculate $I_{1}(x)$ and $I_{2}(y)$. With the aid of Eq. (2), we finally reconstruct the $2 \mathrm{D}$ beam profile, $I(x, y)$. The $2 \mathrm{D}$ intensity distribution functions of the four incident beam profiles we employ for the reconstruction test are listed in Table 2.

In the upper and lower graphs of Fig. 2, we compare the incident and reconstructed beam profiles by going through the above procedure. What we can learn from Fig. 2 is that, if the incident beam profile function is separable in $\mathrm{x}$ and $\mathrm{y}$ directions (Figs. 2(a) and 2(b)), the reconstructed beam profiles are quite accurate. If not (Figs. 2(c) and 2(d)), we see differences between 
Table 2: 2D intensity distribution functions of four incident beam profiles we employ for the reconstruction test.

\begin{tabular}{ll}
\hline Beam profile & 2D intensity distribution function \\
\hline Gaussian & $I(x, y)=\exp \left(-\frac{x^{2}+y^{2}}{180^{2}}\right)$ \\
Elliptical Gaussian & $I(x, y)=\exp \left(-\frac{x^{2}}{2 \times 100^{2}}-\frac{y^{2}}{2 \times 150^{2}}\right)$ \\
Tilted elliptical Gaussian & $I(x, y)=\exp \left(-\frac{(x-y)^{2}}{200^{2}}-\frac{(x+y)^{2}}{300^{2}}\right)$ \\
sech $^{2}$ & $I(x, y)=\operatorname{sech}^{2}\left(\frac{\sqrt{x^{2}+y^{2}}}{154}\right)$ \\
\hline
\end{tabular}
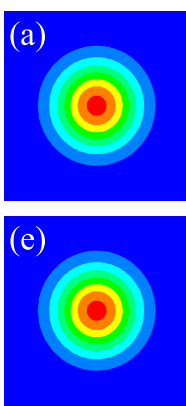

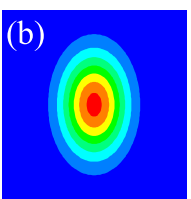

(f)

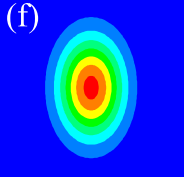

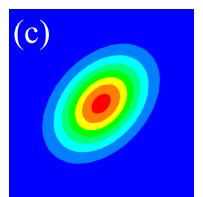

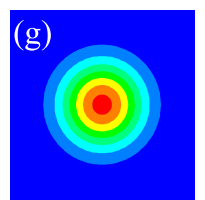

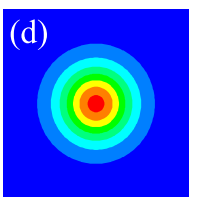

(h)

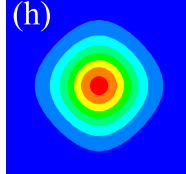

Figure 2: Incident beam profiles defined by the (a) radially symmetric Gaussian, (b) elliptical Gaussian, (c) tilted elliptical Gaussian, and (d) $\operatorname{sech}^{2}$ functions. Corresponding reconstructed 2D beam profiles are given by the lower graphs (e)-(h), respectively.

the incident and reconstructed beam profiles. Knowing this limitation, one can still say that the reconstruction of the $2 \mathrm{D}$ beam profile by assuming the separability of the beam profile function in $\mathrm{x}$ and $\mathrm{y}$ directions would provide us useful information to estimate the spatial quality of the incident beam. This situation is somehow similar to the case of autocorrelation measurement: It never provides us the real pulse duration. Extraction of the pulse duration from the autocorrelation signal is possible only if we may assume that the temporal pulse shape is symmetric with respect to its peak and represented by some function such as Gaussian or hyperbolic secant, etc. Although such limitations exist for the autocorrelation method, nobody doubts that it is a useful technique to estimate the pulse duration of the incident beam. Similar is true for the knife-edge method.

\subsubsection{Data processing}

During the knife-edge scan, uncertainties exist due to some reasons such as the pointing instability of the laser, mechanical inaccuracy of knife-edge 
translation by the step size, etc. These uncertainties will be amplified by the post-scan process of numerically taking the derivatives (Eq. (4)). To reduce the errors arising from those factors, we do not directly take the derivatives from the discrete data points of $S_{1}$ and $S_{2}$. We first look for some appropriate functions by fitting to represent the discrete data points of $S_{1}$ and $S_{2}$ or do the smoothing for the discrete data points of $S_{1}$ and $S_{2}$, and then take the derivatives. As trial functions for the fitting we employ two different functions, i.e., the error (ER) function,

$$
S_{1}(x)=A+B \times \operatorname{erf}\left(\frac{x-x_{0}}{\sqrt{2 \sigma^{2}}}\right),
$$

with $A, B, x_{0}$, and $\sigma$ as free parameters, and double Boltzmann (DB) function,

$$
S_{1}(x)=A_{1}+A_{2} \times\left\{\frac{p}{1+\exp \left[h_{1} \times\left(x_{1}-x\right)\right]}+\frac{1-p}{1+\exp \left[h_{2} \times\left(x_{2}-x\right)\right]}\right\} .
$$

with $A_{1}, A_{2}, p, h_{1}, h_{2}, x_{1}$, and $x_{2}$ as free parameters. The choice of these functions as trial functions are based on the typical shape of $S_{1}$ as illustrated in Fig. 1(b). As for the smoothing, we first add some data points by linear interpolation, and then smooth the discrete $S_{1}$ data points using the 25 points, 4th order Savitzky-Golay method. In Figs. 3(a) and 3(b) we present representative results after the two kinds of fitting and smoothing for $S_{1}$ and $S_{2}$ at $z=150 \mathrm{~mm}$, in which the raw data are shown by the open circles while the results obtained after the ER fitting, DB fitting, and smoothing are shown by the black, red, and blue curves, respectively. Note that the vertical axis of Fig. 3 is in the log scale. In Tables 3 and 4, we summarize the residual sums of squares after the different fitting and smoothing.

By taking the derivatives of $S_{1}$ and $S_{2}$, we obtain the intensity distributions, $I_{1}$ and $I_{2}$, respectively. The results are shown in Figs. 4 and 5 for $I_{1}$ and $I_{2}$, respectively, at eight different positions around the focus. In both Figs. 4 and 5, the open circles represent the derivatives of the raw knife-edge data, while the black, red, and blue curves represent the derivatives of the knife-edge data after the ER fitting, DB fitting, and smoothing, respectively.

Although all the three fitted and smoothed curves in Fig. 3 look similar at first glance, we can see from Tables 3 and 4 that the residual sums of squares after the ER fitting is notably larger than the others, in particular 

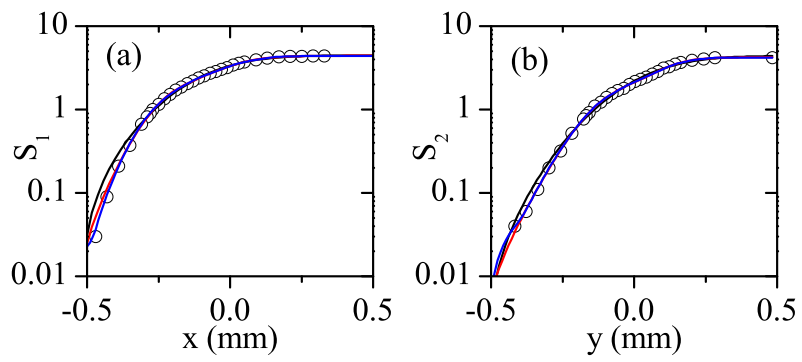

Figure 3: Representative results after the two different fitting and smoothing for the given sets of raw knife-edge data of (a) $S_{1}$ and (b) $S_{2}$ at $z=150 \mathrm{~mm}$. In each graph the raw knife-edge data are represented by the open circles, while the results after the ER fitting, DB fitting, and smoothing are shown by the black, red, and blue curves, respectively. Note that the vertical axes are in the log scale for both graphs.

Table 3: The residual sums of squares after the two different fitting and smoothing for $S_{1}$.

\begin{tabular}{cccc}
\hline $\mathrm{z}(\mathrm{mm})$ & ER fitting & DB fitting & smoothing \\
\hline 150 & 0.0765 & 0.0107 & 0.0057 \\
154 & 0.0316 & 0.0241 & 0.0244 \\
155 & 0.0767 & 0.0163 & 0.0276 \\
156 & 0.0154 & 0.0089 & 0.0197 \\
158 & 0.0198 & 0.0206 & 0.0275 \\
160 & 0.0297 & 0.0153 & 0.0161 \\
162 & 0.0184 & 0.0112 & 0.0117 \\
166 & 0.0181 & 0.0098 & 0.0071 \\
\hline
\end{tabular}

Table 4: Similar to Table 3 but for $S_{2}$.

\begin{tabular}{cccc}
\hline $\mathrm{z}(\mathrm{mm})$ & ER fitting & DB fitting & smoothing \\
\hline 150 & 0.1726 & 0.0156 & 0.0131 \\
154 & 0.0523 & 0.0290 & 0.0256 \\
155 & 0.0399 & 0.0197 & 0.0230 \\
156 & 0.0712 & 0.0279 & 0.0622 \\
158 & 0.0054 & 0.0051 & 0.0290 \\
160 & 0.0309 & 0.0134 & 0.0188 \\
162 & 0.0612 & 0.0171 & 0.0154 \\
166 & 0.0745 & 0.0329 & 0.0143 \\
\hline
\end{tabular}

at the positions far from the focus, i.e., $z=150$ and $166 \mathrm{~mm}$. This means 

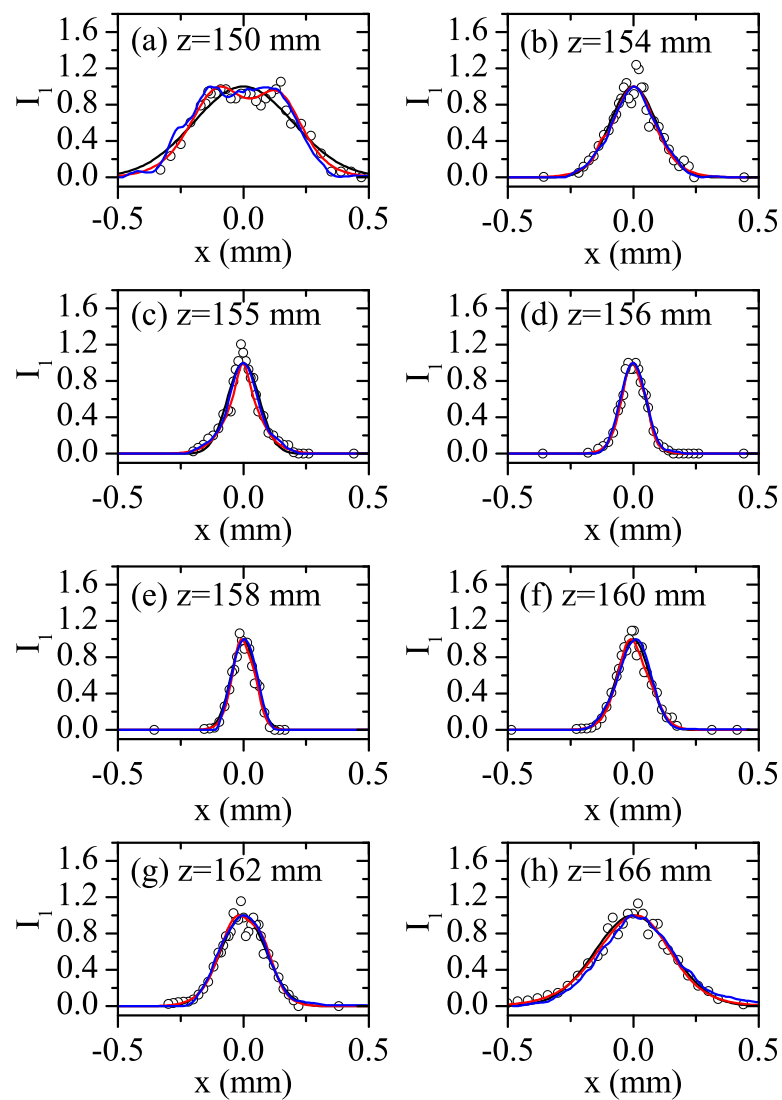

Figure 4: Intensity distributions, $I_{1}$, at different positions around the focus. The open circles represent the derivatives of the raw knife-edge data, while the black, red, and blue curves represent the derivatives of the knife-edge data after the ER fitting, DB fitting, and smoothing, respectively. For graphs (a)-(h), the positions after the $f=150 \mathrm{~mm}$ lens are $150,154,155,156,158,160,162$, and $166(\mathrm{~mm})$, respectively.

that the real beam profile of KU-FEL is not well-represented by the Gaussian function. We emphasize that the precise representation of beam shapes at the positions far from the focus is very important to accurately determine the value of $M^{2}$ (see Fig. 8). Based on this finding, we will only show the 2D beam profiles obtained by the DB fitting and smoothing.

The reconstructed $2 \mathrm{D}$ beam profiles with the data obtained after the DB fitting are shown in Fig. 6 at different positions around the focus. Figure 7 shows the similar results but with the data obtained by the smoothing. 

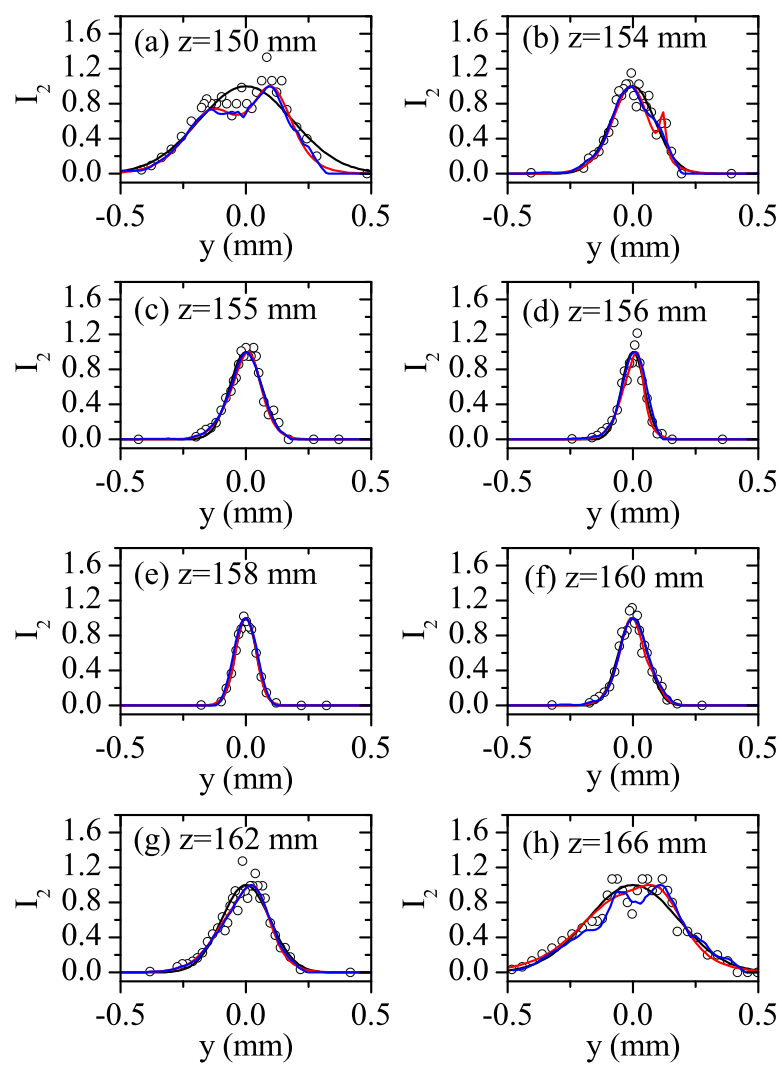

Figure 5: Similar to Fig. 4 but for $I_{2}$.

From Figs. 6 and 7, we find that the 2D beam profiles reconstructed by the two methods are quite similar at any positions: By both methods the beam profiles are almost radially symmetric near the focus, but quite distorted at the far field.

\section{2. $M^{2}$ factor}

To determine the $M^{2}$ factor, we must know the beam diameters at different positions around the focus. An important issue is how to define the beam diameter for non-Gaussian beam. Here we define the beam diameter in terms of $4 \sigma$, which is suggested by the ISO 11146 standard [18]. Such a definition is especially suitable for non-Gaussian beams. The $4 \sigma$ beam diameter in $\mathrm{x}$ direction, $d_{1}^{(4 \sigma)}$, is defined by 

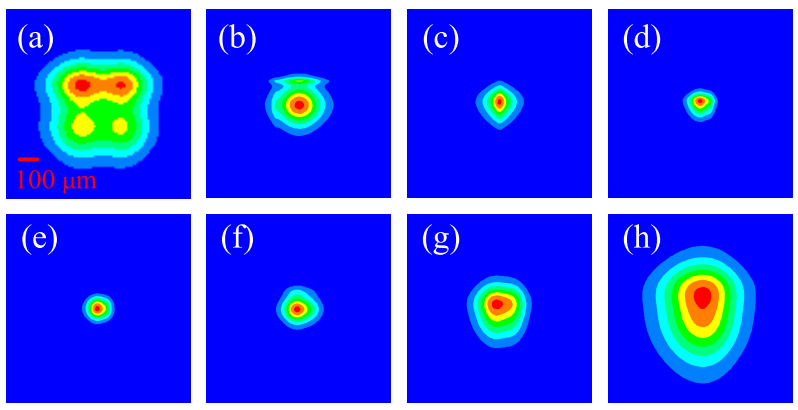

Figure 6: Reconstructed 2D beam profiles at different positions around the focus using the data obtained after the DB fitting. For graphs (a)-(h), the positions after the $f=150$ $\mathrm{mm}$ lens are $150,154,155,156,158,160,162$, and $166(\mathrm{~mm})$, respectively.
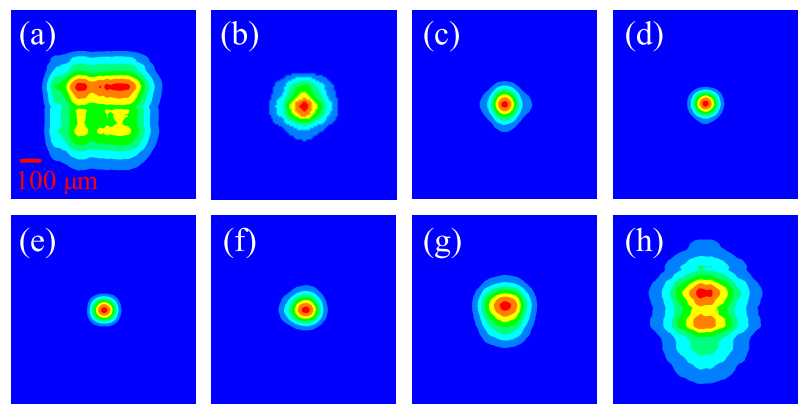

Figure 7: Similar to Fig. 6 but using the data obtained by the smoothing.

$$
d_{1}^{(4 \sigma)}=4 \sqrt{\frac{\int_{-\infty}^{\infty} \int_{-\infty}^{\infty}(x-\bar{x})^{2} I(x, y) d x d y}{\int_{-\infty}^{\infty} \int_{-\infty}^{\infty} I(x, y) d x d y}},
$$

where $\bar{x}$ is the centroid in $\mathrm{x}$ direction, and written as

$$
\bar{x}=\frac{\int_{-\infty}^{\infty} \int_{-\infty}^{\infty} x I(x, y) d x d y}{\int_{-\infty}^{\infty} \int_{-\infty}^{\infty} I(x, y) d x d y} .
$$

The caustic curve of beam diameter as a function of $\mathrm{z}$ under the $\mathrm{x}(\mathrm{y})$ direction scan, i.e., $d_{1(2)}^{(4 \sigma)}(z)$, is obtained by fitting all discrete data points of $d_{1(2)}^{(4 \sigma)}$ (Eq. (7)) at different $\mathrm{z}$ to the following equation: 


$$
d_{1(2)}^{(4 \sigma)}(z)=d_{01(2)} \sqrt{1+\left[\frac{4\left(z-z_{01(2)}\right) \lambda M_{1(2)}^{2}}{\pi d_{01(2)}^{2}}\right]^{2}},
$$

where $d_{01(2)}, z_{01(2)}, \lambda$, and $M_{1(2)}^{2}$ are the beam diameter at the beam waist, position of the beam waist, laser wavelength, and $M^{2}$ factor under the $\mathrm{x}(\mathrm{y})$ direction scan, respectively. During the fitting, $\lambda$ is set to $11 \mu \mathrm{m}$, while $d_{01(2)}$, $z_{01(2)}$, and $M_{1(2)}^{2}$ are left as free parameters. The beam diameters at the beam waist and the divergence angles in $\mathrm{x}$ and $\mathrm{y}$ directions obtained by the fitting are listed in Table 5 for both DB fitting and smoothing methods.

Table 5: The beam diameters at beam waist and divergence angles in $\mathrm{x}$ and $\mathrm{y}$ directions by smoothing and DB fitting method.

\begin{tabular}{ccc}
\hline & Beam diameters $(\mu \mathrm{m})$ & divergence angle $(\mathrm{mrad})$ \\
\hline $\mathrm{x}$, fitting & 181.6 & 82.81 \\
x, smoothing & 186.8 & 81.42 \\
y, fitting & 159.4 & 88.00 \\
y, smoothing & 172.3 & 85.05 \\
\hline
\end{tabular}
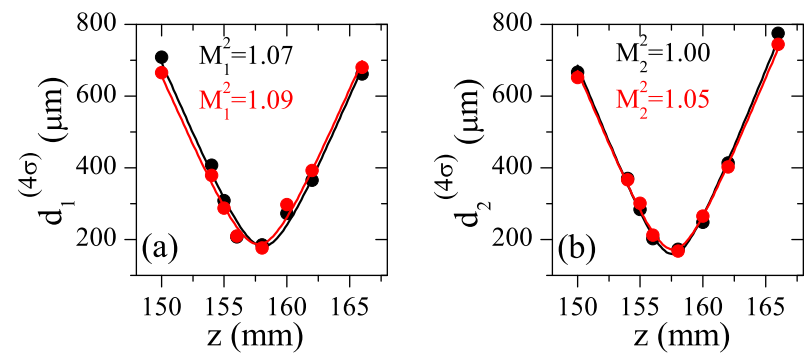

Figure 8: Change of beam diameters in (a) $\mathrm{x}$ and (b) y directions as a function of position. Dots are the beam diameters in $\mathrm{x}$ and y directions, $d_{1}^{(4 \sigma)}$ and $d_{2}^{(4 \sigma)}$, obtained with Eq. (7), while the caustic curves are obtained by fitting the $d_{1(2)}^{(4 \sigma)}$ with Eq. (9). In both graphs, the results obtained by the DB fitting method are shown by black curves, while the results obtained by the smoothing method are shown by red curves. The $M_{1(2)}^{2}$ factors in x and y directions obtained by the two methods are written in the graphs with the corresponding colors.

Figure 8 shows the change of beam diameters $d_{1}^{(4 \sigma)}$ and $d_{2}^{(4 \sigma)}$ in both $\mathrm{x}$ (Fig. 8(a)) and y (Fig. 8(b)) directions. In Fig. 8, the dots are the mea- 
sured data while the black and red curves represent the fitted results by the DB function and smoothing, respectively. We find that the $M^{2}$ factors determined by both fitting and smoothing methods are very similar and very close to unity, which implies that the KU-FEL beam has very good focusing quality.

To confirm the correctness of our results, a simple calculation is performed to estimate the beam diameter at the beam waist and the divergence angle after focus under our experimental condition. It is known that, when the distance between the beam waist and the lens is much larger than the focal length of the lens, the beam waist diameter of a laser beam after the focus is

$$
d_{0}^{\prime}=\frac{4 M^{2} \lambda f}{\pi d}
$$

and the divergence angle after the focus is

$$
\theta^{\prime}=\frac{d}{f}
$$

where $\lambda, f$, and $d$ refer to the wavelength, focal length of the lens, and the beam diameter on the lens, respectively. For our case, $\lambda=11 \mu \mathrm{m}, f=150$ $\mathrm{mm}, d=14 \mathrm{~mm}$, and $M^{2}=1.1$, we obtain $d_{0}^{\prime}=165.1 \mu \mathrm{m}$ and $\theta^{\prime}=93.33$ mrad, respectively, which are very similar to our results shown in Table 5 with the difference of about $10 \%$. After these estimations, we are more confident that the results reported in this paper are correct.

\section{BURN PATTERN TEST}

As a cross-check, we also undertake the burn pattern experiment. The KU-FEL beam is focused to an acrylic plate with a thickness of $2 \mathrm{~mm}$ by the $f=100 \mathrm{~mm}$ lens. For each maropulse with $\sim 9.5 \mathrm{~mJ}$ energy, we take a burn pattern at a different position around the focus. Figures $9(\mathrm{a})-9(\mathrm{~g})$ show the burn patterns taken at the positions of $z=102,103,104,105,106,107$, and 108 (mm), respectively.

Since the lenses with different focal lengths are used for the knife-edge and burn pattern experiments, we cannot say anything more than qualitative to compare the both data. But from our experience, the dependence of the beam shape on the focal lens in very weak, if not zero. From Fig. 9, we find that the beam shape is almost radially symmetric near the focus (Figs. 9 (c)-9(e)), but distorted at the far field (Figs. 9(a), 9(b), 9(f), and 9(g)). 

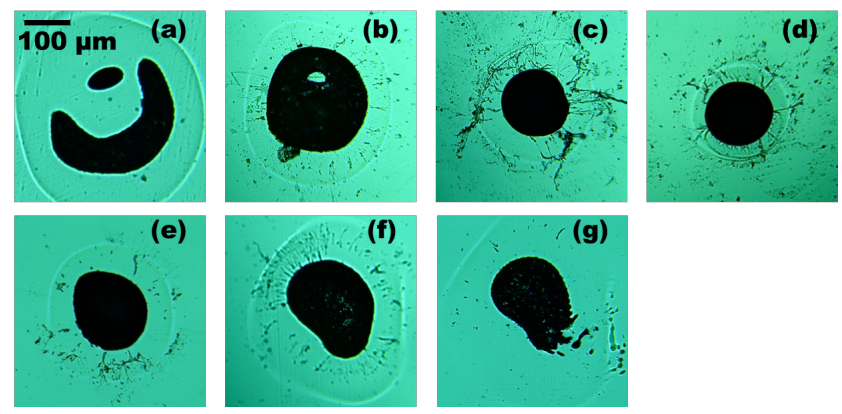

Figure 9: Burn patterns at the positions of $z=102,103,104,105,106,107$, and 108 (mm), which correspond to graphs (a)-(g), respectively. The macropulse energy is $\sim 9.5 \mathrm{~mJ}$.

This trend we see in Fig. 9 is consistent with the findings by the knife-edge experiment we have described in the previous section.

\section{CONCLUSIONS}

We have carried out the characterization of KU-FEL beam at $11 \mu \mathrm{m}$ by the knife-edge method. The knife-edge data we have obtained suggest that the KU-FEL beam has a non-Gaussian shape. To represent the nonGaussian beam shape we have employed the two methods; fitting to some simple analytical function and smoothing of the knife-edge data. It turned out that both methods work equally well. From the knife-edge data after such data processings, we have reconstructed the $2 \mathrm{D}$ beam profiles at different positions around the focus. The reconstructed $2 \mathrm{D}$ beam profiles are almost radially symmetric near the focus, but distorted at the far field. By employing the definition of the beam diameter in terms of $4 \sigma$, which is suggested by the ISO 11146 standard for non-Gaussian beams [18], we have determined the $M^{2}$ factors to be about 1.1 in both $\mathrm{x}$ and y directions. This suggests that the KU-FEL beam has very good focusing quality. The measured $M^{2}$ value is consistent with our recent experimental findings that we have demonstrated the high damage threshold and focusability of KU-FEL pulse gated by a plasma mirror with nanosecond switching pulses [6].

\section{acknowledgments}

This work is supported by a Grant-in-aid for Scientific Research from the Ministry of Education and Science of Japan. 


\section{References}

[1] D. D. L. Elias, J. Madey, G. Ramian, H. Schwettman, T. Smith, First operation of a free-electron laser, Phys. Rev. Lett 38 (1977) 892-894.

[2] P. G. O'Shea, H. P. Freund, Free-electron lasers: status and applications, Science 292 (5523) (2001) 1853-1858.

[3] H. Ohgaki, T. Kii, K. Masuda, H. Zen, S. Sasaki, T. Shiiyama, R. Kinjo, K. Yoshikawa, T. Yamazaki, Lasing at 12 mum mid-infrared freeelectron laser in kyoto university, Jpn. J. Appl. Phys. 47 (2008) 8091.

[4] Y. Qin, H. Zen, X. Wang, T. Kii, T. Nakajima, H. Ohgaki, Pulse duration and wavelength stability measurements of a midinfrared freeelectron laser, Opt. Lett. 38 (7) (2013) 1068-1070.

[5] X. Wang, T. Nakajima, H. Zen, T. Kii, H. Ohgaki, Single-shot spectra of temporally selected micropulses from a mid-infrared free-electron laser by upconversion, Opt. Lett. 37 (24) (2012) 5148-5150.

[6] X. Wang, T. Nakajima, H. Zen, T. Kii, H. Ohgaki, Damage threshold and focusability of mid-infrared free-electron laser pulses gated by a plasma mirror with nanosecond switching pulses, Appl. Phys. Lett. 103 (2013) 191105.

[7] P. B. Chapple, Beam waist and $\mathrm{m} 2$ measurement using a finite slit, Opt. Eng. 33 (7) (1994) 2461-2466.

[8] P. Shayler, Laser beam distribution in the focal region, Appl. Opt. 17 (1978) 2673.

[9] J. Arnaud, W. Hubbard, G. Mandeville, B. De la Claviere, E. Franke, J. Franke, Technique for fast measurement of gaussian laser beam parameters, Appl. Opt. 10 (12) (1971) 2775-2776.

[10] J. M. Khosrofian, B. A. Garetz, Measurement of a gaussian laser beam diameter through the direct inversion of knife-edge data, Appl. Opt. 22 (21) (1983) 3406-3410.

[11] B. E. Newnam, R. W. Warren, R. L. Sheffield, W. E. Stein, L. M. T, J. S. Fraser, J. C. Goldstein, J. E. Sollid, T. A. Swann, J. M. Watson, 
C. A. Brau, Optical performance of the los alamos free-electron laser, IEEE J. of Quantum Electron. 21 (7) (1985) 867-881.

[12] S. V. Benson, J. Gubeli, M. Shinn, Mode distortion measurements on the jefferson lab ir fel, Nuclear Instruments and Methods in Physics Research Section A: Accelerators, Spectrometers, Detectors and Associated Equipment 483 (1) (2002) 434-439.

[13] S. Quabis, R. Dorn, M. Eberler, O. Glöckl, G. Leuchs, The focus of light-theoretical calculation and experimental tomographic reconstruction, Appl. Phys. B 72 (1) (2001) 109-113.

[14] M. A. de Araújo, R. Silva, E. de Lima, D. P. Pereira, P. C. de Oliveira, Measurement of gaussian laser beam radius using the knife-edge technique: improvement on data analysis, Applied optics 48 (2) (2009) 393396.

[15] M. González-Cardel, P. Arguijo, R. Díaz-Uribe, Gaussian beam radius measurement with a knife-edge: a polynomial approximation to the inverse error function, Appl. Opt. 52 (16) (2013) 3849-3855.

[16] J. Chalupský, J. Krzywinski, L. Juha, V. Hájková, J. Cihelka, T. Burian, L. Vyšín, J. Gaudin, A. Gleeson, M. Jurek, A. R. Khorsand, D. Klinger, H. Wabnitz, R. Sobierajski, M. Störmer, K. Tiedtke, S. Toleikis, Spot size characterization of focused non-gaussian x-ray laser beams, Opt. Express 18 (26) (2010) 27836-27845.

[17] B. Flöter, P. Juranić, S. Kapitzki, B. Keitel, K. Mann, E. Plönjes, B. Schäfer, K. Tiedtke, Euv hartmann sensor for wavefront measurements at the free-electron laser in hamburg, New J. Phys. 12 (2010) 083015 .

[18] ISO 11146:1999, Lasers and laser-related equipment - Test methods for laser beam parameters $\mathrm{C}$ Beam widths, divergence angle and beam propagation factor. 